\title{
THE EFFECT OF HYALURONIC ACID ON NUCLEUS PULPOSUS EXTRACELLULAR MATRIX PRODUCTION THROUGH HYPOXIA- INDUCIBLE FACTOR-1A TRANSCRIPTIONAL ACTIVATION OF CD44 UNDER HYPOXIA
}

\author{
F. Zhang ${ }^{1, \$}$, X. $\mathrm{Liu}^{2, \S}, \mathrm{B} . \mathrm{Li}^{1}$, Z. $\mathrm{Li}^{3}, \mathrm{~S} . \mathrm{Grad}^{3}$, D. Chen ${ }^{4}, \mathrm{M} . \mathrm{Gao}^{1,2, *}$ and S. $\mathrm{Liu}^{1,2}$ \\ ${ }^{1}$ Innovation Platform of Regeneration and Repair of Spinal Cord and Nerve injury, Department of \\ Orthopaedic Surgery, The Seventh Affiliated Hospital of Sun Yat-sen University, Shenzhen, China \\ ${ }^{2}$ Guangdong Provincial Key Laboratory of Orthopaedics and Traumatology, \\ The First Affiliated Hospital of Sun Yat-sen University, Guangzhou, China \\ ${ }^{3} \mathrm{AO}$ Research Institute Davos, Davos, Switzerland \\ ${ }^{4}$ Laboratory of Bone Tissue Engineering, Beijing Laboratory of Biomedical Materials, Beijing Research \\ Institute of Orthopaedics and Traumatology, Beijing JiShuiTan Hospital, Beijing, China \\ $\S$ These authors contributed equally to this work
}

\begin{abstract}
Intervertebral disc degeneration (IDD) is the leading cause of low-back pain. Implantation of hyaluronic acid (HA) is potentially a therapeutic strategy for IDD, but its pharmacological effects and mechanism under hypoxic conditions remain unclear. In this study, the expression of extracellular matrix genes and proteins were enhanced in nucleus pulposus cells (NPCs) in the presence of HA under hypoxic condition, as shown by real-time reverse transcription-polymerase chain reaction, immunofluorescence staining, and dimethylmethylene blue assays. Moreover, the expression of CD44 was increased in the presence of both $\mathrm{HA}$ and hypoxia compared to either alone. Using a bioinformatic database, hypoxia inducible factor- $1 \alpha$ (HIF-1 $\alpha$ ), a key transcription factor in the hypoxic condition, was found to have 4 predicted binding sites on the CD44 promoter. CD44 expression was significantly increased by treatment with cobalt chloride or dimethyloxalylglycine. Over-expression of HIF-1 $\alpha$ in NPCs significantly up-regulated the expression of CD44. The binding site of HIF- $1 \alpha$ in the CD44 promoter region, was identified by promoter truncation experiments and chromatin immunoprecipitation assays. Taken together, these results indicated that hypoxic conditions positively potentiated the ability of NPCs matrix synthesis in the presence of HA, which correlated with the increasing CD44 expression by HIF-1 $\alpha$ transcriptional activation.
\end{abstract}

Keywords: Intervertebral disc degeneration, hypoxia inducible factor- $1 \alpha, \mathrm{CD} 44$, hyaluronic acid, gene regulation.

*Address for correspondence: Manman Gao, Ph.D., Innovation Platform of Regeneration and Repair of Spinal Cord and Nerve injury, Department of Orthopaedic Surgery, The Seventh Affiliated Hospital of Sun Yat-sen University, Shenzhen, China / Guangdong Provincial Key Laboratory of Orthopaedics and Traumatology, The First Affiliated Hospital of Sun Yat-sen University, Guangzhou, China.

Telephone number: +0755 23242436 Email: gaomanm@mail2.sysu.edu.cn

Copyright policy: This article is distributed in accordance with Creative Commons Attribution Licence (http://creativecommons.org/licenses/by-sa/4.0/).

\begin{tabular}{|c|c|c|c|}
\hline \multicolumn{3}{|c|}{ List of Abbreviations } & $\begin{array}{l}\text { the surface of most B lymphocytes and } \\
\text { differentiating neuroblasts }\end{array}$ \\
\hline $5^{\prime} \mathrm{UTR}$ & $5^{\prime}$ untranslated region & CD44 & a main cell surface receptor of HA \\
\hline ADAMTS4 & a disintegrin and metalloproteinase & ChIP & chromatin immunoprecipitation \\
\hline & with thrombospondin motifs 4 & COL II & collagen II \\
\hline ANOVA & analysis of variance & DAPI & 4',6-diamidino-2-phenylindole \\
\hline BSA & bovine serum albumin & $\mathrm{dH}_{2} \mathrm{O}$ & distilled water \\
\hline CA12 & carbonic anhydrase 12 & DMEM & Dulbecco's modified Eagle's medium \\
\hline CCK & cell counting kits & DMMB & dimethylmethylene blue \\
\hline $\mathrm{CCN} 2$ & cellular communication network factor 2 & DMOG & dimethyloxalylglycine \\
\hline CD24 & a sialoglycoprotein expressed at & ECL & enhanced chemiluminescence \\
\hline
\end{tabular}




\begin{tabular}{|c|c|}
\hline ECM & extracellular matrix \\
\hline EDTA & ethylenediaminetetraacetic acid \\
\hline FBS & foetal-bovine serum \\
\hline GAG & glycosaminoglycan \\
\hline GAPDH & $\begin{array}{l}\text { glyceraldehyde 3-phosphate } \\
\text { dehydrogenase }\end{array}$ \\
\hline HA & hyaluronic acid \\
\hline HABP2 & HA-binding protein 2 \\
\hline HIF-1 $\alpha$ & hypoxia-inducible factor- $1 \alpha$ \\
\hline HRE & hypoxia-responsive element \\
\hline HRP & horseradish peroxidase \\
\hline IDD & intervertebral-disc degeneration \\
\hline IF & immunofluorescence \\
\hline IL-1R1 & interleukin 1 receptor, type I \\
\hline IVD & intervertebral disc \\
\hline MyD88 & myeloid differentiation factor 88 \\
\hline NP & nucleus pulposus \\
\hline NPCs & NP cells \\
\hline OCT & optimal cutting temperature \\
\hline Over-HIF-1 $\alpha$ & HIF-1 $\alpha$ over-expression construct \\
\hline PBS & phosphate-buffered saline \\
\hline PCR & polymerase chain reaction \\
\hline PHD & prolyl-hydroxylases \\
\hline PMSF & phenylmethylsulphonyl fluoride \\
\hline RIPA & radio-immunoprecipitation assay \\
\hline RT-qPCR & real-time reverse transcription PCR \\
\hline SD & standard deviation \\
\hline SDC4 & syndecan4 \\
\hline SDS & sodium dodecyl sulphate \\
\hline TBST & tris-buffered saline with polysorbate 20 \\
\hline TSS & transcription start sites \\
\hline
\end{tabular}

\section{Introduction}

Low-back pain is a common health problem that affects up to $80 \%$ of individuals during their lifetime, imposing enormous health and economic burdens on society (Takahashi et al., 2008). IDD is considered to be a primary cause of low-back pain (Vergroesen et al., 2015). Current therapies for IDD range from rehabilitation and medications to invasive surgical interventions. All of them have limited efficacy and can neither address the underlying degeneration nor restore the native function of the IVD (An et al., 2003). For this reason, there is a compelling need to develop a therapeutic approach with improved outcomes.

HA is a major non-sulphated GAG present in the ECM of the IVD. In NP tissue, HA plays a critical role in ECM production, cell migration and phenotypic maintenance, acting as the backbone of proteoglycan aggregates that contain aggrecan molecules (Chung et al., 2008). As IDD progresses, the molecular weight and concentration of HA in the NP decrease, which results in further disc degeneration through enhancement of matrix degradation, inflammatory response, and reduction of water retention in the discs (Quero et al., 2013).

With the development of tissue engineering technology, implantation of HA-based hydrogel is a novel biological treatment for early stage IDD. Various HA hydrogels have been investigated for their potential to replace and regenerate the
NP through reducing inflammation response and nociceptive behaviour (Isa et al., 2015; Mohd et al., 2018), promoting ECM synthesis (Kim et al., 2015), and restoring biomechanical properties (Zhou et al., 2014). Furthermore, it has recently been shown that HA hydrogels could promote stem cell differentiation to NP-like cells and improve the efficacy of stem cells for NP regeneration (Chen et al., 2019; Peroglio et al., 2013). Some studies have explored the mechanism by which HA promotes NP regeneration. For example, HA could modulate the inflammatory receptor of IL-1R1, MyD88 and neurotrophin expression (Isa et al., 2015), or downregulate the catabolic marker ADAMTS4 (Kazezian et al., 2017). However, these studies assessing the biological effects and molecular mechanisms of HA for NP regeneration, have been performed under standard normoxic conditions, which is not representative of the physiological hypoxic environment in the intervertebral disc.

CD44, plays an important role in regulating multiple cellular functions (Ishida et al., 1997). It has previously been shown that hypoxia potentiated the anabolic effects of $\mathrm{HA}$, including the redifferentiation of articular chondrocytes and synthesis of ECM, through enhancing CD44 expression and binding affinity (Ichimaru et al., 2016). Furthermore, previous research suggested that hypoxia could enhance the expression of CD44 via HIF-1 $\alpha$ (Liang et al., 2017). Based on the bioinformatics databases JASPAR (Web ref. 1) and Promoter 2.0 Prediction Server (Web ref. 2), HIF-1 $\alpha$ binding sites were found in the human CD44 gene promoter region, which means that HIF$1 \alpha$ may transcriptionally activate CD44, resulting in enhanced CD44 expression (Fornes et al., 2020).

Based on these above findings, the hypothesis that hypoxic conditions could enhance the effect of HA for NP regeneration through HIF-1 $\alpha$ transcriptional activation of CD44 is proposed. In this study, the aim was to determine the transcriptional activation of CD44 by HIF- $1 \alpha$ and explore the ability of NPCs proliferation and matrix synthesis under hypoxic conditions in the presence of HA. The therapeutic potential of HA for the treatment of IDD in hypoxic condition was demonstrated.

\section{Materials and Methods}

\section{Human NPCs culture and treatment}

NP tissues were obtained during discectomy surgery from 10 patients with lumbar-degenerative diseases (Table 1). All procedures were performed in accordance with the guidance and approval of the research ethics committee of the $7^{\text {th }}$ Affiliated Hospital of Sun Yat-sen University, Shenzhen, Guangdong, China. The tissue of the central portion of NP was washed 3 times with PBS (Gibco) to eliminate blood, and cut into $1 \mathrm{~mm}^{3}$ fragments. Subsequently, NP tissue was digested for 30 min with $0.25 \%$ trypsinEDTA (Gibco) followed by $0.25 \%$ collagenase type II for $4 \mathrm{~h}$ at $37^{\circ} \mathrm{C}$, with agitation. After filtration 
through a $70 \mu \mathrm{m}$ nylon mesh to remove tissue debris, the NPCs were isolated by centrifugation at $1000 \times g$ for $5 \mathrm{~min}$. The NPCs were maintained in DMEM/nutrient mixture F12 (Gibco) supplemented with $10 \%$ FBS (Gibco) and $50 \mu \mathrm{g} / \mathrm{mL}$ Primocin (InvivoGen, San Diego, CA, USA), and incubated in a humidified atmosphere with $5 \% \mathrm{CO}_{2}$ at $37^{\circ} \mathrm{C}$. The anti-microbial agents were replaced with $1 \%$ penicillin/streptomycin (Gibco) after the cells reached P2. The cells of passage 3, 4 and 5 were used for the experiments.

For the hypoxia treatment, cells were maintained in a Hypoxia Work Station (Panasonic, Osaka, Japan) with a mixture of $2 \% \mathrm{O}_{2}, 5 \% \mathrm{CO}_{2}$, and $93 \% \mathrm{~N}_{2}$ for 24-72 h. Due to the longer half-life and potential to increase the amount of endogenous HA and exhibit anti-inflammatory activity, high molecular weight HA (MW = 1,800-2,200 kDa) was chosen according to a previous study (Chen et al., 2013). Considering that HA degrades when exposed to high temperature, ultraviolet rays or ultrasound (Snetkov et al., 2020), high molecular weight HA was placed in an ampoule and the air removed, then sterilised at $121^{\circ} \mathrm{C}$ for $15 \mathrm{~min}$, to minimise molecular weight loss. NPCs were cultured in 6-well plate (Corning, NY, USA) $\left(10^{5}\right.$ cells/ well) with complete DMEM/F12 containing $2 \mathrm{mg} /$ $\mathrm{mL}$ high molecular weight HA (Freda, Jinan, China) for $72 \mathrm{~h}$ under normoxic or hypoxic conditions. To induce hypoxic stress, NPCs were treated with 100, 200 or $400 \mu \mathrm{mol} / \mathrm{L} \mathrm{CoCl}_{2}$ (Sigma, St. Louis, MO, USA) for $24 \mathrm{~h}$. To stabilise HIF-1 $\alpha$ by inhibition of PHD function, NP cells were treated with 0.5 or $1 \mathrm{mmol} / \mathrm{L}$ DMOG (MCE, Monmouth Junction, NJ, USA) for $24 \mathrm{~h}$ (Fujita et al., 2012).

\section{Plasmids and cell transfection}

Human CD44 promoter reporter constructs from nucleotide - 2934 to ATG (2934-pCD 44) and control reporter vector were developed by Genecoepia (Rockville, MD, USA). The plasmid with HIF-1 $\alpha$ over-expression construct or control vector were purchased from Genecoepia (Rockville). 2803-pCD44, 908-pCD44, 442-pCD44, 339-pCD44 were generated by restriction enzyme digestion of 2934-pCD44, using the primers listed in Table 2.
Lipofectamine LTX \& PLUS Reagent (Invitrogen, Carlsbad, CA,USA) was used in the transient transfection according to the manufacturer's instructions. Briefly, $10^{5}$ cells/well NPCs were transferred to 6-well plates (Corning) in DMEM/F12 with $10 \%$ FBS before transfection. When the cells were at a confluence of $70-80 \%$, the plasmid with HIF-1 $\alpha$ over-expression construct or control vector was diluted in Opti-MEM ${ }^{\circledR}$ reduced serum medium (Invitrogen), and mixed with lipofectamine reagent to prepare plasmid DNA-lipid complexes, then added to cells. Following incubation for $3 \mathrm{~d}$ at $37^{\circ} \mathrm{C}$ under normoxic or hypoxic conditions, the cells were harvested, and the protein was extracted for the subsequent Western blot analysis.

\section{RT-qPCR}

Total RNA was extracted from the treated NPCs using an RNAeasy ${ }^{\mathrm{TM}}$ animal RNA isolation Kit (Beyotime, Shanghai, China) according to the manufacturer's protocol. Reverse transcription was performed using PrimeScript ${ }^{\mathrm{TM}}$ RT Master Mix (TaKaRa, Shiga, Japan) according to the manufacturer's instruction. Real-time PCR was performed using PowerUp $\mathrm{p}^{\mathrm{TM}}$ SYBR $^{\mathrm{TM}}$ Green Master Mix (Applied Biosystems, Foster City, CA, USA) in CFX96 Real-Time System (Bio-Rad Laboratories, Hercules, CA, USA). The total volume $(10 \mu \mathrm{L})$ of each PCR reaction contained $5 \mu \mathrm{L}$ SYBR Green Master Mix, $2 \mu \mathrm{L}$ RNase-free $\mathrm{dH}_{2} \mathrm{O}, 2 \mu \mathrm{L}$ cDNA, and $0.5 \mu \mathrm{L}$ of each forward and reverse primer (Table 3). The housekeeping gene GAPDH was used for normalisation, and the relative expression of each target gene was determined using the $2^{-\Delta \Delta \mathrm{Ct}}$ method.

\section{Protein extraction and Western blot analysis}

Following treatment, NPCs were placed on ice immediately and washed 3 times with ice-cold PBS. Cells were lysed by RIPA lysis buffer (Boster, Wuhan, China) containing $1 \%$ PMSF (Boster) and $1 \%$ Halt $^{\mathrm{TM}}$ Protease Inhibitor Cocktail (Thermo Fisher Scientific, Waltham, MA, USA), followed by sonication (Sonics CVX130, Newtown, MA, USA) for $30 \mathrm{~s}$. Then, the protein was isolated by centrifugation at $12,000 \times g$ for $10 \mathrm{~min}$ at $4{ }^{\circ} \mathrm{C}$. Protein extracts were resolved by electrophoresis on NuPAGE ${ }^{\mathrm{TM}} 4-12 \%$ Bis-Tris

Table 1. Clinical patient characteristics.

\begin{tabular}{|c|c|c|c|c|c|}
\hline Patient & Age & Gender & Disc level & Degeneration grade & Experiment \\
\hline 1 & 40 & female & L4/5 & III & Cell culture \\
\hline 2 & 66 & female & L4/5 & III & Cell culture \\
\hline 3 & 36 & male & L4/5 & III & Cell culture \\
\hline 4 & 63 & male & L4/5 & III & Cell culture \\
\hline 5 & 53 & male & L3/4 & V & RT-qPCR, IF \\
\hline 6 & 46 & female & L5/S1 & II & RT-qPCR, IF \\
\hline 7 & 70 & male & L4/5 & IV & RT-qPCR, IF \\
\hline 8 & 24 & male & L4/5 & III & RT-qPCR, IF \\
\hline 9 & 73 & male & L4/5 & IV & RT-qPCR, IF \\
\hline 10 & 46 & male & L4/5 & II & RT-qPCR, IF \\
\hline
\end{tabular}


Table 2. Primer sequences used for vector construction.

\begin{tabular}{|c|c|c|c|}
\hline Vector name & Organism & Sense primer & Antisense primer \\
\hline 2803-pCD44 & Homo sapiens & ACTAGTTTCAGGTGGGAGTTACGGGT & CATATGCATGCAGTAAGCACTTTAAA \\
\hline 908-pCD44 & Homo sapiens & ACTAGTGGATGGGCGGATGGAAGGAT & CAGCTGCTCGCCAGCGGTGC \\
\hline 442-pCD44 & Homo sapiens & ACTAGTGCCAGCGGGAGAAGAAAGCC & CAGCTGCTCGCCAGCGGTGC \\
\hline 339-pCD44 & Homo sapiens & ACTAGTGGCAGCCCCGATTATTTACA & CAGCTGCTCGCCAGCGGTGC \\
\hline
\end{tabular}

Table 3. Primer sequences used for RT-qPCR experiments.

\begin{tabular}{|c|c|c|c|}
\hline Gene transcript & Organism & Sense primer & Antisense primer \\
\hline CD44 & Homo sapiens & TGGCATCCCTCTTGGCCTTGG & TGAGACTTGCTGGCCTCTCCGT \\
\hline Aggrecan & Homo sapiens & TCCCCTGCTATTTCATCGAC & CCAGCAGCACTACCTCCTTC \\
\hline Collagen II & Homo sapiens & GGAGCAGCAAGAGCAAGGAGAA & AGCAGGCGTAGGAAGGTCATCT \\
\hline GAPDH & Homo sapiens & CGGAGCCAAAAGGGTCATCA & GGGGGGCTAAGCAGTTGGTG \\
\hline
\end{tabular}

gels (Invitrogen) and transferred by electroblotting to PVDF membrane (Invitrogen). The membrane was rinsed in water and blocked with $5 \%$ non-fat dry milk in TBST (Leagene, Beijing, China) for $2 \mathrm{~h}$ at room temperature with constant agitation. The membrane was then incubated overnight at $4{ }^{\circ} \mathrm{C}$ in TBST with antibodies against CD44 (1: 2,000, ab157107, Abcam, Cambridge, UK), HABP2 (1 : 2,000, ab181837, Abcam), $\beta$-actin (1 : 2,000, Cell Signaling Technology, Danvers, MA, USA). After washing the membrane 3 times with TBST, goat anti-mouse HRP (1 : 4000, ab205719, Abcam) or goat anti-rabbit HRP ( $1: 4000$, ab205718, Abcam) was added and incubated at room temperature for $2 \mathrm{~h}$. Following washing 3 times with TBST, the immunoblots were visualised using an ECL reagent (Beyotime). The protein band densities were quantified using Image J software and presented as relative level to $\beta$-actin. The experiment was repeated 3 times.

\section{IF staining}

Human NP tissues were fixed in $4 \%$ paraformaldehyde for $24 \mathrm{~h}$ and dehydrated in graded sucrose solutions. Tissues were embedded in OCT compound (Sakura, Torrance, CA, USA) and then cut into $10 \mu \mathrm{m}$-thick cryosections. The sections were permeabilised in PBS containing $0.3 \%$ Triton X-100 (BioFroxx, Shanghai, China) for $30 \mathrm{~min}$, and then blocked in PBS containing $5 \%$ BSA (BioFroxx) and $0.1 \%$ Triton X-100 (BioFroxx) for $1 \mathrm{~h}$. Subsequently, sections were incubated overnight at $4{ }^{\circ} \mathrm{C}$ in dilutions of the antibodies against CD44 (1: 500, ab157107, Abcam) and/or HIF$1 \alpha$ (1:200, ab1, Abcam). After thoroughly washing the sections with TBST, sections were incubated with Alexa Fluor-488 conjugated anti-rabbit and/or Fluor-594 conjugated anti-mouse secondary antibody (Jackson ImmunoResearch Inc., West Grove, PA, USA) at $1: 300$ dilution for $1 \mathrm{~h}$ at room temperature, and then washed with TBST before counterstaining with DAPI (Abcam) for $5 \mathrm{~min}$. The staining was visualised using a laser confocal microscope (LSM 880 with Airyscan, Zeiss Microscopy GmbH, Jena, Germany).

For cultured cell immunofluorescence staining, NPCs were seeded on glass coverslips. After the treatment, cells were washed with PBS, fixed in $4 \%$ paraformaldehyde for $20 \mathrm{~min}$, then permeabilised and blocked with PBS containing $5 \%$ BSA and $0.3 \%$ Triton $\mathrm{X}-100$ for $1 \mathrm{~h}$ at room temperature. Subsequently, cells on coverslips were incubated with antibodies against CD44 (1 : 500, ab157107, Abcam), HIF-1 $\alpha$ (1:200, ab1, Abcam), COL II (1:200, Cell Signaling Technology), aggrecan (1 : 200, ab3778, Abcam). The following immunostaining protocols were identical to cryosections as described above. The ImageJ software was used to semi-quantitative analysis of the fluorescence intensity (Jensen et al., 2013).

\section{Cell viability analyses}

To evaluate NPC proliferation in the presence of HA under hypoxic conditions, the CCKs (MCE) were used. Briefly, NPCs were seeded in 24-well plates $\left(2 \times 10^{4}\right.$ cells/well) in the presence or absence of HA under normoxic or hypoxic conditions. Following culturing for $72 \mathrm{~h}, 50 \mu \mathrm{L}$ CCK solution was added to cells, and monitored spectrophotometrically at $450 \mathrm{~nm}$ after incubation for $2 \mathrm{~h}$.

\section{Biochemical analyses}

To quantify the amount of GAG in NPCs, a DMMB assay was performed using GAG detection kits (Genmed Scientifics Inc., Arlington, MA, USA) according to the manufacturer's instructions. Briefly, the soluble GAG was extracted with high-salt solution and combined with DMMB dye. After adding a propanol dissociation solution, the purple dye was released and monitored spectrophotometrically (SynergyH1, BioTek, Winooski, VT, USA). The total amount of GAG was calculated by comparison with standard samples provided by the GAG detection kits $(50 \mathrm{ng} / \mu \mathrm{L})$ and normalised to the total amount of DNA (NanoDrop One, Thermo Fisher Scientific, MA, USA) as measured by the spectrophotometer in the same sample (Stone et al., 1994).

\section{Dual-luciferase reporter assay}

To confirm the direct activation of CD44 by HIF-1 $\alpha$, dual-luciferase reporter assay was performed. HEK 293 cells were seeded in 96 -well plates $\left(5 \times 10^{3}\right.$ cells $/$ 
well) in DMEM/F12 with $10 \%$ FBS, $1 \mathrm{~d}$ before transfection. Cells were transfected with HIF-1 $\alpha$ overexpression construct and human CD44 promoter reporter construct (2934-pCD44, 2803-pCD44, 908pCD44, 442-pCD44, 339-pCD44) or control vector. After incubation for $48 \mathrm{~h}$, the firefly and Renilla luciferase activities were detected by dual-luciferase reporter assay system (Promega, Madison, WI, USA). The relative luciferase activity was determined by the ratio of firefly luciferase : Renilla luciferase activity (internal control) (Gao et al., 2018).

\section{ChIP}

To confirm HIF- $1 \alpha$ activated CD44 expression by the predicted site, ChIP assays were performed using the ChIP Assay Kit (Beyotime) according to the manufacturer's protocol. Briefly, following culture in $100 \mathrm{~mm}$ dishes under hypoxic conditions for $3 \mathrm{~d}$, NPCs were incubated in $1 \%$ formaldehyde solution at $37^{\circ} \mathrm{C}$ for $10 \mathrm{~min}$, and glycine solution was added to stop the reaction. After washing in PBS with $1 \%$ PMSF and lysis with SDS buffer, the cells was treated by sonication (Sonics CVX130, city, state, USA) to cut genomic DNA. ChIP grade antibody against HIF-1 $\alpha$ (1:200, ab1, Abcam) was used to bind the proteinDNA complex. Following washing and unlinking by $5 \mathrm{~mol} / \mathrm{L} \mathrm{NaCl}$, the DNA fragments were purified using a DNA Purification Kit (Beyotime). Finally, the purified DNA was amplified by PCR with the primers listed in Table 4 . The predicted binding site 1 and 2 were too close to design primers separately, so the designed primers 'ChIP site 12 ' contained the predicted binding site 1 and 2 .

\section{Statistical analysis}

Statistical analysis was performed using SPSS 20.0 statistical software. Quantitative data were presented as means \pm SD. Data distribution was checked with Shapiro-Wilk normality test, and non-normally distributed data were examined by using the Mann-Whitney test. Differences among the groups were identified by $t$-test or one-way ANOVA. In all analyses, $p<0.05$ was considered statistically significant.

\section{Results}

\section{Expression of HIF-1 $\alpha$ and CD44 in NPCs and in NP tissues}

Human NP tissues were divided into the mild degeneration group (II -III grade) and the severe degeneration group (IV-V grade), according to the Pfirrmann grading system (Pfirrmann et al., 2001). The immunofluorescence staining and RT-qPCR results showed that CD44 expression progressively decreased from the mild degeneration to severe degeneration group $(p<0.05)$ (Fig. 1a,b). In NPCs, the confocal images showed the co-expression of HIF-1 $\alpha$ and CD44 (Fig. 1c). To explore the distribution of HIF- $1 \alpha$ and CD44 in NP tissues, immunofluorescence co-staining was performed and showed that HIF-1 $\alpha$ and CD44 co-expressed in NP tissues (Fig. 1d).

\section{Hypoxia with HA potentiates NPCs proliferation and the synthesis of ECM}

To determine the metabolic effects of exogenous HA and hypoxia on the ECM, human NPCs were cultured for $72 \mathrm{~h}$ in the presence or absence of HA under normoxic or hypoxic conditions. In the presence of HA under hypoxic condition, the cell proliferation was significantly enhanced $(p<0.01)$ (Fig. 2a), and the total GAG amount was increased as observed by DMMB assays $(p<0.05)$ (Fig. $2 \mathbf{b})$, in contrast to in the absence of HA under normoxic condition. Immunofluorescence staining results showed that the level of COL II and aggrecan expression were significantly higher in NPCs treated with HA under hypoxic conditions than in the absence of HA under normoxic condition $(p<0.05)$ (Fig. 2c-f). The aggrecan mRNA expression was increased in the presence of HA under hypoxic condition, but the change was not statistically significant (Fig. $2 \mathbf{h})(p>0.05)$, and the COL II mRNA expression showed no obvious change (Fig. 2g) $(p>0.05)$.

\section{The expression of CD44 and HABP2 were enhanced by hypoxia with HA}

To explore the mechanism by which HA potentiates the synthesis of ECM under hypoxic conditions, CD44 and HABP2 expression were detected. The expression of CD44 protein in NPCs increased with HA treatment and hypoxia. Importantly, the expression of CD44 protein increased in the presence of both HA and hypoxia compared to either of them alone. The level of HABP2 was increased in the presence of HA under hypoxic conditions, relative to the level in the absence of HA under normoxic condition $(p<0.05)$ (Fig. 3a-d). The gene expression of CD44 also appeared to be higher in the presence of HA under hypoxic condition than relative to its level in the absence of HA under normoxic conditions, but the difference was not statistically significant $(p>0.05)$ (Fig. 3e).

Table 4. Primer sequences used for ChIP assays.

\begin{tabular}{|c|c|c|}
\hline Gene transcript & Sense primer & Antisense primer \\
\hline ChIP site 12 & ATCCCTGGAGGTTGCATGAG & ATGACTCAAAGAGCCGCCC \\
\hline ChIP site 3 & CATGTGTGTGGAGAGAGGTGC & GTGGAAAGAGGGAGAGCTCATT \\
\hline ChIP site 4 & GCCAAAGGCTGAACCCAATG & GTGCTCTGCTGAGGCTGTAA \\
\hline
\end{tabular}



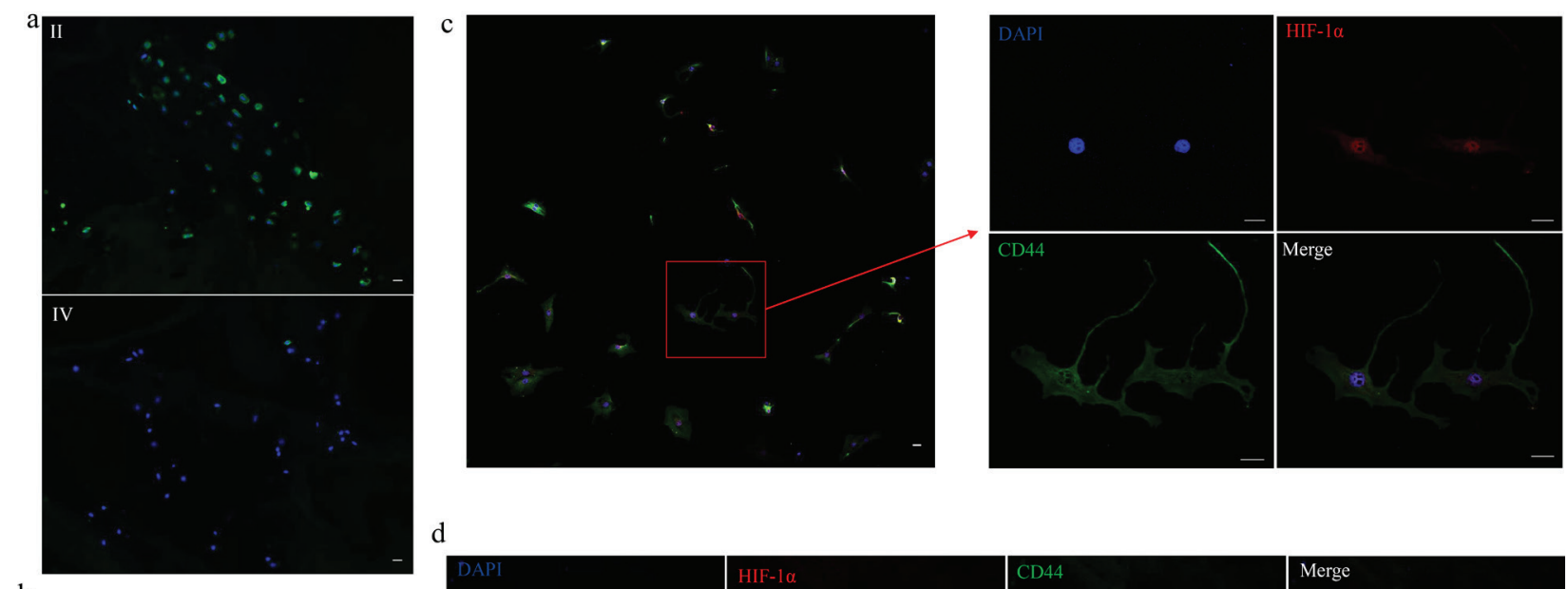

$\mathrm{b}$
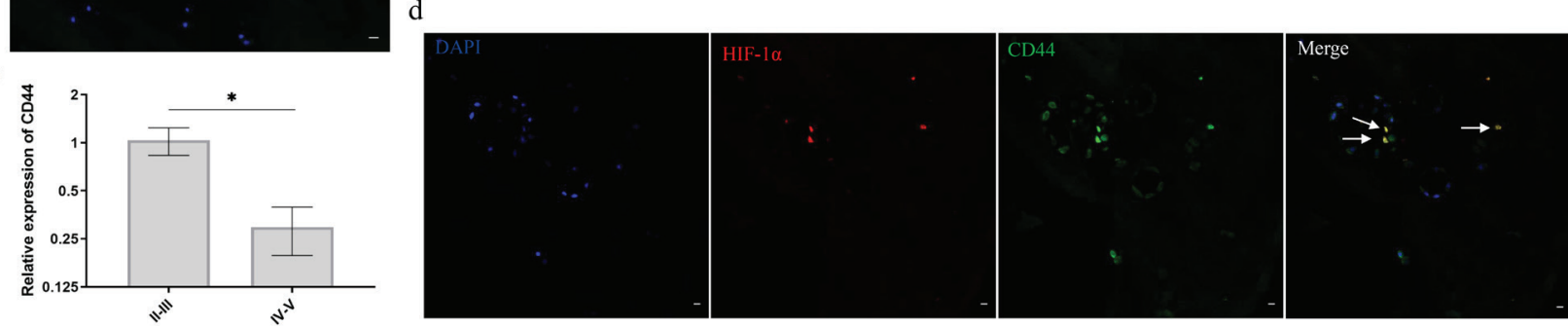

Fig. 1. Expression of HIF-1 $\alpha$ and CD44 in NPCs and in NP tissues. Human NP tissues were divided in the mild degeneration group (II -III grade) and the severe degeneration group (IV-V grade) according to the Pfirrmann grading system. (a) The CD44 protein expression between 2 groups was analysed by immunofluorescence staining. (b) The CD44 mRNA expression between 2 groups was detected by RT-qPCR. (c) The passaged cells obtained from the mild degeneration disc, were cultivated in hypoxic condition for $3 \mathrm{~d}$. The immunofluorescent co-staining results showed the expression pattern of HIF-1 $\alpha$ and CD44. CD44 (green); HIF- $1 \alpha$ (red); nuclei, DAPI (blue). (d) The immunofluorescent co-staining results showed the expression pattern of HIF-1 $\alpha$ and CD44 in the mild degeneration NP tissues. Data represent average values of 3 independent experiments, error bars represent $\mathrm{SD},{ }^{*} p<0.05$. Scale bar: $10 \mu \mathrm{m}$.

a
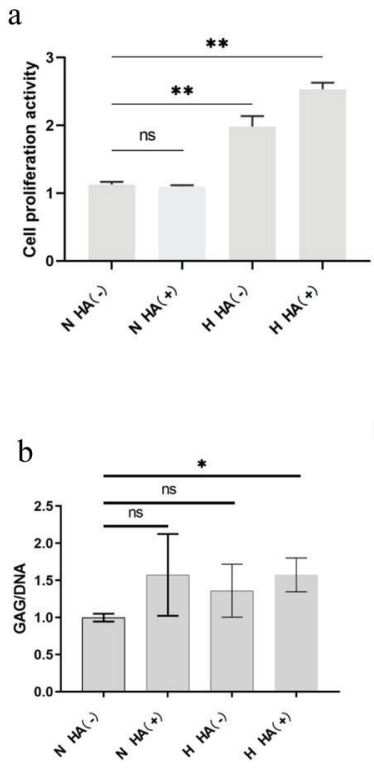
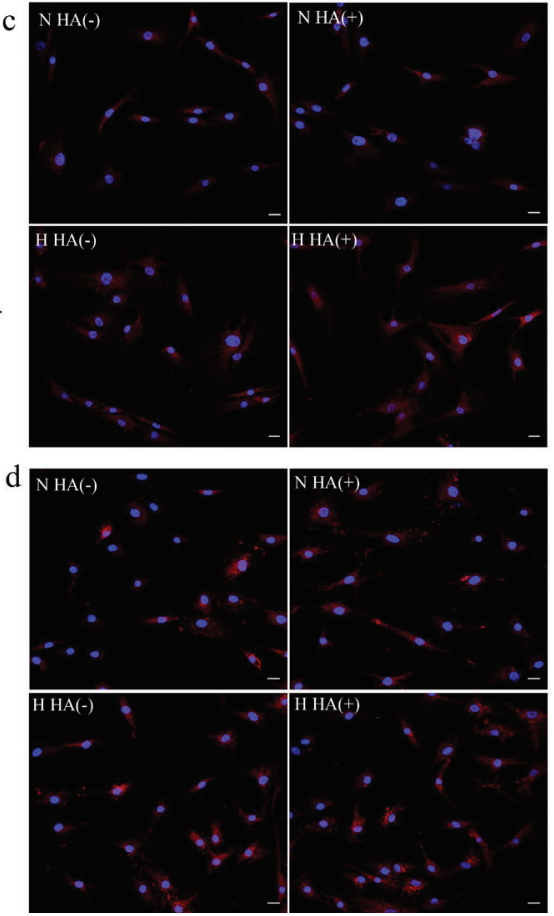
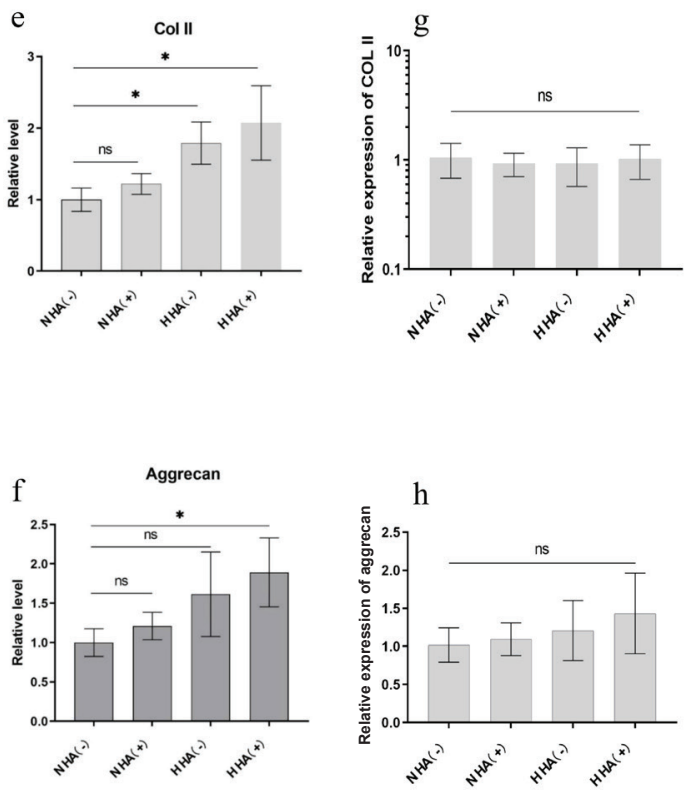

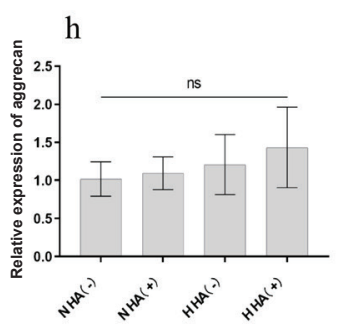

Fig. 2. Hypoxia with HA potentiates NPC proliferation and synthesis of ECM. Human NPCs were cultured for $72 \mathrm{~h}$ in the presence $(+)$ or absence $(-)$ of HA under normoxic $(\mathrm{N})$ or hypoxic $(\mathrm{H})$ conditions. (a) The cell proliferation was tested using CCK-8. (b) The total amount of GAG in each sample was quantified by DMMB assay and normalised relative to DNA level. The COL II (c) and aggrecan (d) expression were detected by immunofluorescence staining. (e,f) Semi-quantification of 3 repetitions of the immunofluorescence staining results. The COL II (g) and aggrecan (h) mRNA expression was detected by RT-qPCR. Data represent average values of 3 independent experiments, normalised to the value of the control group [N HA (-)], error bars represent SD. ns, not significant, ${ }^{*} p<0.05,{ }^{* *} p<0.01$. Scale bar: $20 \mu \mathrm{m}$. 
a

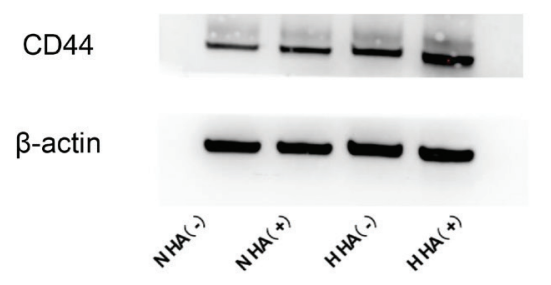

$\mathrm{b}$

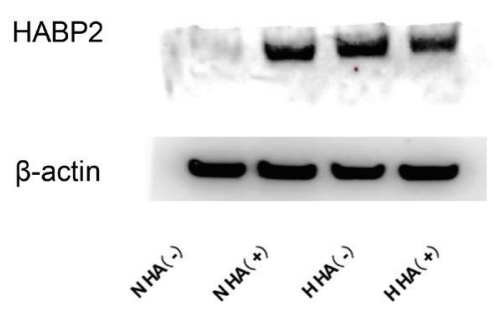

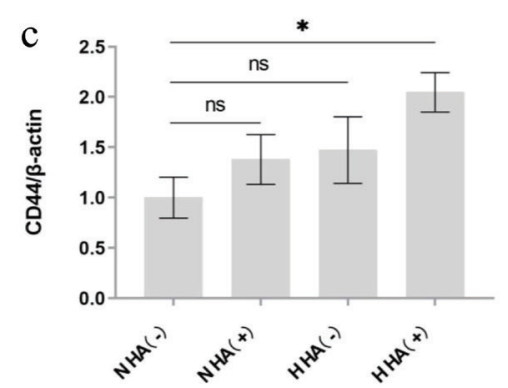
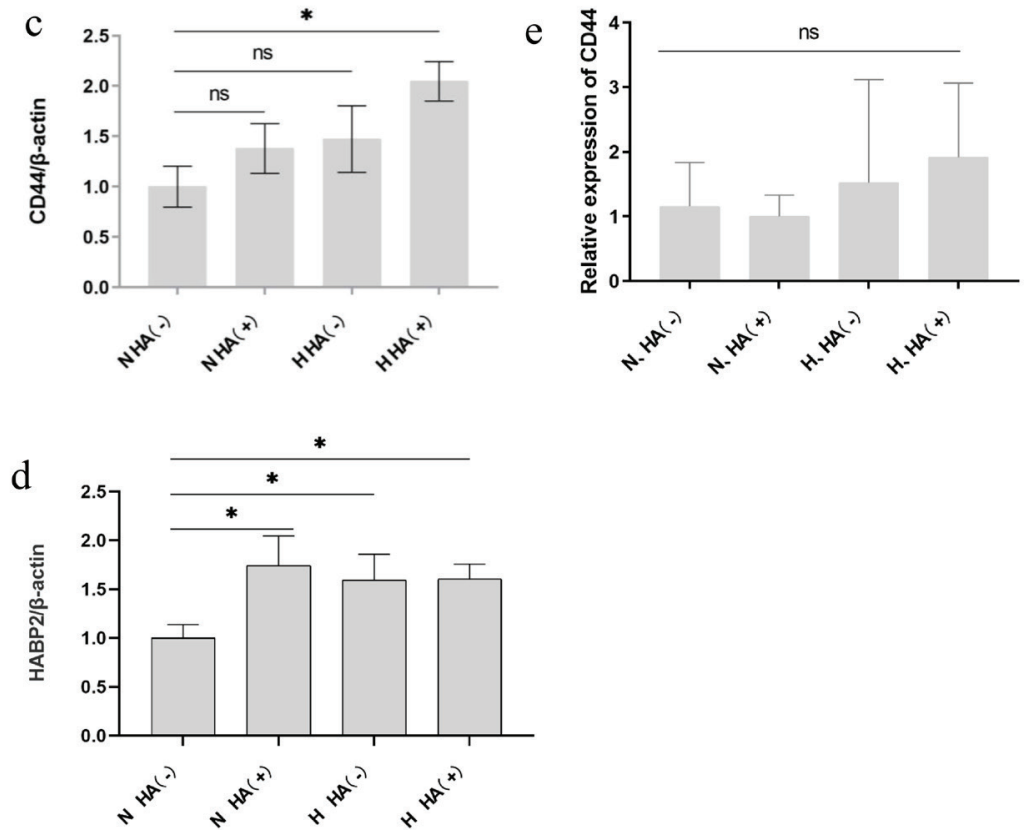

Fig. 3. The expression of CD44 and HABP2 was enhanced by hypoxia with HA. Human NPCs were cultured for $72 \mathrm{~h}$ in the presence $(+)$ or absence $(-)$ of HA under normoxic $(\mathrm{N})$ or hypoxic $(\mathrm{H})$ conditions. The protein levels of CD44 (a) and HABP2 (b) were detected by Western blotting, and 3 repetitions of the western blot results were analysed quantitatively by Image J software (c,d). (e) The relative mRNA expression of CD44 was detected by RT-qPCR. Mean \pm SD, ${ }^{*} p<0.05$.

a

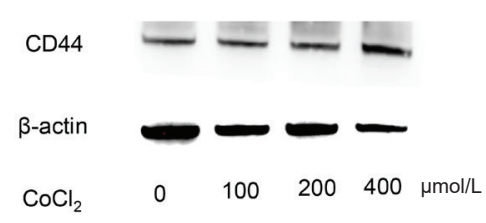

$\mathrm{c}$

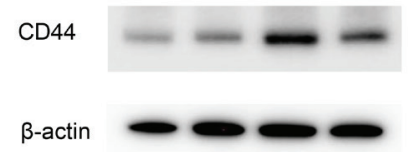

$\beta$-actin

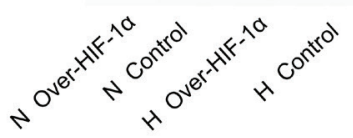

e

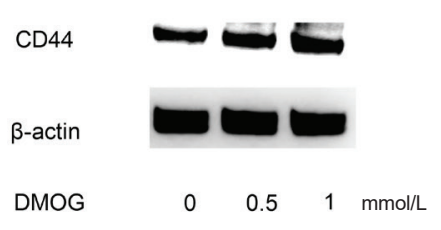

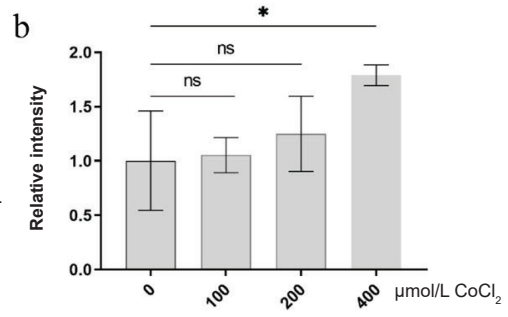

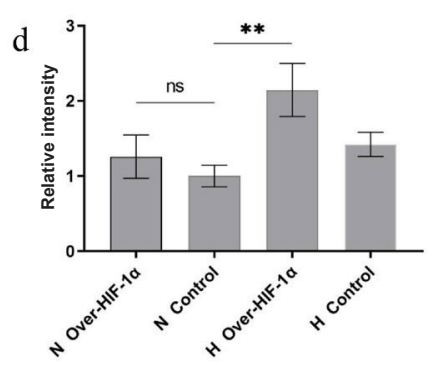

f

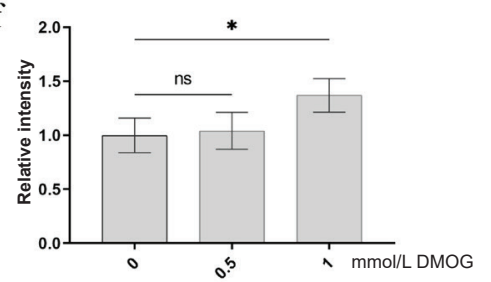

g

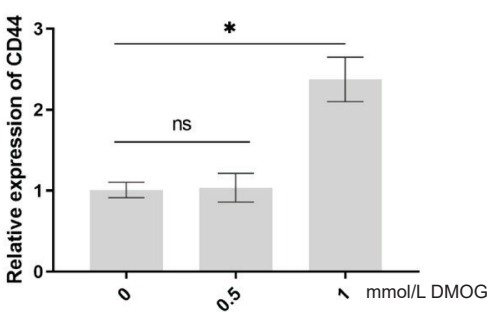

h

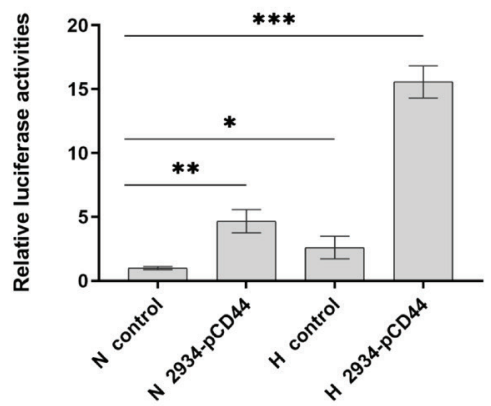

Fig. 4. CD44 expression was induced by hypoxia and regulated by PHD/HIF-1 $\alpha$. (a,b) NPCs were treated with $\mathrm{CoCl}_{2}$ for $24 \mathrm{~h}$, the protein level of CD44 was detected by Western blotting. (c,d) NPCs were transfected with Over-HIF-1 $\alpha$ or basic vector (control) and cultured under normoxic $(\mathrm{N})$ or hypoxic $(\mathrm{H})$ conditions for $72 \mathrm{~h}$, the protein level of CD44 was detected by western blotting, the group ( $\mathrm{N}$ control) was control group. NPCs were treated with DMOG for $24 \mathrm{~h}$, the expression of CD44 protein $(\mathbf{e}, \mathbf{f})$ and mRNA $(\mathbf{g})$ were detected by western blotting and RT-qPCR. (h) 293T cells were transfected with human CD44 promoter reporter vector (2934-pCD44) or control reporter vector (control) and cultured under normoxic $(\mathrm{N})$ or hypoxic $(\mathrm{H})$ conditions for $48 \mathrm{~h}$, the relative luciferase activities were detected. Data represent average values of 3 independent experiments. Error bars represent SD. ns, not significant, ${ }^{*} p<0.05,{ }^{* *} p<0.01,{ }^{* * *} p<0.001$. 


\section{CD44 expression was induced by hypoxia and regulated by PHD/HIF-1 $\alpha$}

To examine the relationship between CD44 and relative hypoxic conditions in NPCs, NPCs were treated by $\mathrm{CoCl}_{2}$, chemical reagent known to induce hypoxic stress, whereby the CD44 expression was dose-dependently induced by $\mathrm{CoCl}_{2}$ (Fig. $4 \mathbf{a}, \mathbf{b}$ ). To investigate the effect of HIF- $1 \alpha$ regulation on CD44 expression, the Over-HIF-1 $\alpha$ or the control vector were transfected into NPCs for $72 \mathrm{~h}$ under normoxic or hypoxic conditions. The results showed that overexpression of HIF- $1 \alpha$ in NPCs up-regulated CD44 expression, especially under the hypoxic conditions $(p<0.01)$ (Fig. 4c,d). Further, to determine that the CD44 expression was regulated by PHD/HIF-1 $\alpha$, NPCs were treated by DMOG, a PHD inhibitor. Thereby, the mRNA expression and protein level of CD44 were increased, as observed by western blotting and RT-qPCR (Fig. 4e-g). Dual-luciferase reporter assay results showed that hypoxic conditions could significantly increase the luciferase activity in 293T cells transfected with the human CD44 promoter reporter vector, when compared with the control group transfected with control reporter vector $(p<0.001)$ (Fig. 4h).

\section{Activation of CD44 promoter by HIF-1 $\alpha$}

To gain further insight into the mechanisms of transcriptional regulation of CD44 by HIF- $1 \alpha$, the first $3 \mathrm{~kb}$ of the Human CD44 promoter were obtained from the UCSC Genome Browser (Web ref. 3). TSS were predicted by the Promoter 2.0 Prediction Server, results showed that a "highly likely prediction" site located position - 300 (Fig. 5a) (Web ref. 2). Next, the first $3 \mathrm{~kb}$ of the human CD44 promoter was scanned for the presence of HREs using the JASPAR core database (Web ref. 1), at an $80 \%$ profile score threshold, 4 putative HRE sites were reported, as shown in Fig. 5b. Luciferase reporter constructs with the 2934 bp promoter sequence of CD44 were constructed for measuring the effect of hypoxia and HIF- $1 \alpha$ on promoter activity. Dual-luciferase reporter assays showed that HIF- $1 \alpha$ significantly increased the activity of the human CD44 promoter fragments (Fig. 5c). To further define the binding sites, serial deletion analysis of the Human CD44 promoter showed that luciferase activity of 2803-pCD44, 908-pCD44, 442pCD44, 339-pCD44 were significantly higher than the control group $(p<0.01)$ (Fig. $5 d$ d). ChIP results showed that HIF-1 $\alpha$ could bind any of the 4 predicted sites (Fig. 5e).

\section{Discussion}

Previous studies revealed that hypoxic conditions enhanced the anabolic effects of HA in articular chondrocytes (Ichimaru et al., 2016). However, the role of hypoxia for HA treatment of NPCs has not been determined. In the current study, the expression of ECM genes and proteins were enhanced in the presence of HA under hypoxic condition, which was correlated with the increasing CD44 expression. Furthermore, it was found that CD44 was a hypoxiasensitive gene, and the regulation was dependent on the PHD - HIF- $1 \alpha$ system, especially transcriptional activation by HIF- $1 \alpha$.

High molecular weight HA has been reported to promote ECM synthesis, increase matrix content, and enhance mechanical properties under normoxic
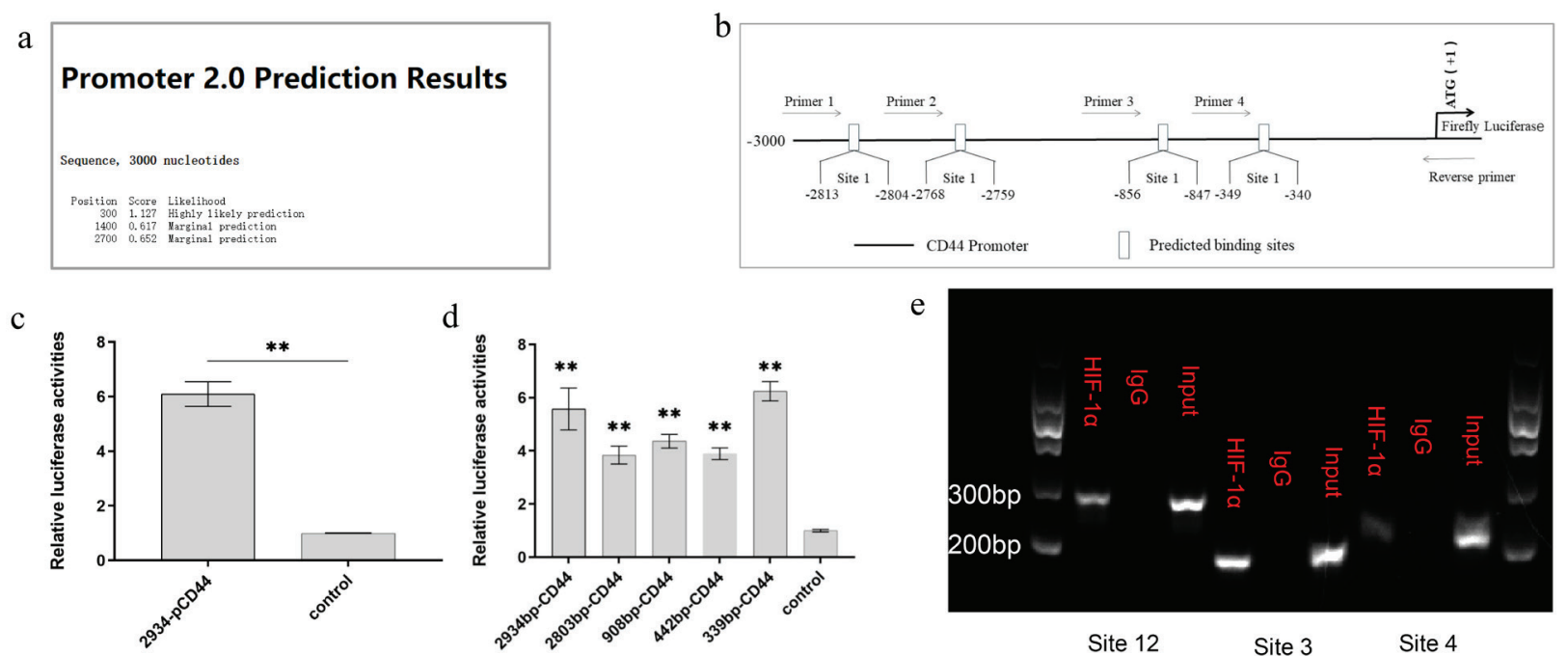

Fig. 5. Activation of CD44 promoter by HIF-1 $\alpha$. (a) Prediction of CD44 transcription starting sites using the database of the Promoter 2.0 Prediction Server. (b) Schematic diagram showing the luciferase construct with predicted binding sites of HIF-1 $\alpha$ on the CD44 promoter acquired from the JASPAR database. (c-d) Relative luciferase activities in 293 T cells transfected with Over-HIF-1 $\alpha$ and Human CD44 promoter vector (2934pCD44, 2803-pCD44, 908-pCD44, 442-pCD44, 339-pCD44) or control reporter vector (control). (e) ChIP results of NPCs showing the regulating sites of HIF-1 $\alpha$ on CD44. Each experiment was repeated 3 times. ${ }^{* *} p<0.01$. 
conditions (Kazezian et al., 2017; Kim et al., 2015), which related to high expression of CD44 receptor after HA treatment (Isa et al., 2015). In addition, compared with normoxia, hypoxia has been reported to promote ECM synthesis and enhance the expression of genes encoding NP ECM (Feng et al., 2013), which through HIF-1 $\alpha$ regulate some target genes, such as CCN2 and CA12 (Chen et al., 2016; Tran et al., 2013). Although HA and hypoxia have been individually reported to enhance matrix metabolism in NPCs, their combined effects remain unclear. In this study, although there was no significant increase in the expression of aggrecan and COL II mRNA, the GAG content and the protein of COL II, aggrecan and HABP2 were increased in the presence of HA under hypoxic condition compared to normoxic conditions in the absence of HA. These findings suggested that hypoxic conditions positively potentiated the ability of NPCs matrix synthesis in the presence of $\mathrm{HA}$, which was consistent with previous results in articular chondrocytes (Ichimaru et al., 2016).

As the most important receptor for hyaluronic acid, CD44 plays an important role in regulating cell adhesion, growth, survival, migration, differentiation and HA metabolism (Ponta et al., 2003). Moreover, CD44 is important in intervertebral disc formation by anchoring hyaluronan to the cells (Stevens et al., 2000). It was found that the expression of CD44 gene and protein were decreased in the severely degenerated disc, as shown by RT-qPCR and immunofluorescence staining, which means that the lack of expression of CD44 may be linked to IDD. Previous studies have revealed that HA promotes NP regeneration by enhancing the expression of CD44 and binding to CD44 in NPCs (Isa et al., 2015). In this study, the expression of CD44 was increased in the presence of both HA and hypoxia compared to either alone, suggesting that the possible mechanism of hypoxic conditions potentiate the ability of NPCs matrix synthesis was by enhancing the expression of CD44.

HIF-1 $\alpha$, a key transcription factor in hypoxic condition, plays an important role in promoting cell survival, matrix synthesis, and regulating energy metabolism, in the disc under hypoxic condition (Choi et al., 2015; Risbud et al., 2006). Through HIF$1 \alpha$, hypoxia controls a host of factors, such as SDC4 (Fujita et al., 2014), CA12 (Chen et al., 2016) and CD24 (Liu et al., 2018), which contribute to maintain homeostasis of NP and protect against degeneration in hypoxic condition of the disc. Although there was no evidence to conclude that HIF- $1 \alpha$ could enhance the expression of CD44 in NPCs, several researchers have shown that expression of CD44 correlated with hypoxia-induced gene signatures in tumours. Previous research found that CD44 expression was associated with hypoxia-induced gene signatures and poor survival in human glioblastoma multiforme (Pietras et al., 2014). In breast cancer, HIF-1 $\alpha$ increased the expression of CD44 molecules and the percentage of CD44 positive cells under hypoxia condition, and a higher expression of CD44 was observed in the hypoxic tumour regions in vivo (Krishnamachary et al., 2012). In addition, hypoxia enhanced CD44 expression via HIF-1 $\alpha$ to promote cell proliferation and invasion, in gastric cancer cells (Liang et al., 2017).

In the current study, it was shown that CD44 expression was induced by hypoxia and selectively regulated by the PHD - HIF- $1 \alpha$ system in NP cells. Firstly, to induce hypoxic stress, NPCs were treated with $\mathrm{CoCl}_{2}$, a chemical inducer of HIF-1 $\alpha$, and CD44 expression was dose-dependently induced. We further found that CD44 expression was increased by transfection with the HIF-1 $\alpha$ over-expression plasmid in the hypoxic condition. However, HIF-1 $\alpha$ did not modulate CD44 expression under normoxia, which may be related to rapid degradation of HIF-1 $\alpha$ in normoxic condition (Fujita et al., 2012). Subsequently, PHDs was inhibited by DMOG, a PHD inhibitor, and it was found that CD44 expression was dose-dependently induced, which demonstrated the importance of PHDs in HIF- $1 \alpha$-induced CD44 expression.

HIF-1 $\alpha$ regulates several genes by binding to a specific site in the promoter region, termed HRE (Semenza, 2000). Based on the Promoter Prediction server and JASPAR core database, 4 HREs were predicted in the human CD44 promoter. Dualluciferase reporter assay results demonstrated that hypoxia and HIF-1 $\alpha$ could regulate the expression of CD44. Moreover, the promoter truncation experiments and ChIP assays further confirmed that HIF- $1 \alpha$ could transcriptionally activate CD44 expression by binding to any of the predicted sites. Taken together, the results clearly indicated that CD44 was a hypoxia-sensitive gene, and the regulation was dependent on the PHD - HIF1 system, especially the transcriptional activation by HIF-1 $\alpha$. After truncating all 4 predicted binding sites, the activity of luciferase was still markedly higher than that of the control group, which indicated that HIF-1 $\alpha$ could activate CD44 expression by binding to the left sequence, most of which belongs to the 5' UTR. Several studies have found that $5^{\prime}$ UTR functions as a regulatory element in certain genes (Bonnet-Magnaval et al., 2016; Gao et al., 2018). More detailed studies would be carried out to identify the regulatory elements in the $5^{\prime}$ UTR of CD44 in the future.

This study had some limitations. Only the synergistic effect of hypoxia and HA on the synthesis of ECM in 2D cell culture was explored, which is not close to the physiological environment in the disc. To address this limitation, the effect of HA will further be tested in organ models or in animal models. Another limitation to this study was that a HA concentration of $1 \mu \mathrm{mol} / \mathrm{L}$ was selected, because most therapeutic pharmaceutical formulations are administered at this concentration (Rayahin et al., 2015), which may be not the optimal concentration in the hypoxic microenvironment of the disc. 


\section{Conclusions}

Together, results from this study clearly indicated that hypoxic conditions enhanced the ability of matrix synthesis of NPCs in the presence of HA, which was nicely correlated with up-regulated $C D 44$ expression upon transcriptional activation of HIF-1 $\alpha$. Therefore, genetic or pharmacological modulation of HIF-1 $\alpha / C D 44$ activity combined with high molecular weight HA may be a novel therapeutic approach to degenerative disc disease.

\section{Acknowledgements}

This study was funded by the National Natural Science Foundation of China (81772400, 31900583, 51873069), Fundamental Research Funds for the Central Universities (19ykzd05), the Natural Science Foundation of Guangzhou City (201704030082, 201807010031), Foundation of Shenzhen Committee for Science and Technology Innovation (JCYJ20190809142211354, GJHZ20180929160004704), Sanming Project of Medicine in Shenzhen (SZSM201911002), Beijing Municipal Health Commission (BMHC-2019-9; BMHC-2018-4; PXM2020_026275_000002).

\section{References}

An H, Boden SD, Kang J, Sandhu HS, Abdu W, Weinstein J (2003) Summary statement: emerging techniques for treatment of degenerative lumbar disc disease. Spine (Phila Pa 1976) 28: S24-S25.

Bonnet-Magnaval F, Philippe C, Van Den Berghe L, Prats H, Touriol C, Lacazette E (2016) Hypoxia and ER stress promote Staufen1 expression through an alternative translation mechanism. Biochem Biophys Res Commun 479: 365-371.

Chen P, Ning L, Qiu P, Mo J, Mei S, Xia C, Zhang J, Lin X, Fan S (2019) Photo-crosslinked gelatinhyaluronic acid methacrylate hydrogel-committed nucleus pulposus-like differentiation of adipose stromal cells for intervertebral disc repair. J Tissue Eng Regen Med 13: 682-693.

Chen S, Fang XQ, Wang Q, Wang SW, Hu ZJ, Zhou ZJ, Xu WB, Wang JY, Qin A, Fan SW (2016) PHD/HIF1 upregulates CA12 to protect against degenerative disc disease: a human sample, in vitro and ex vivo study. Lab Invest 96: 561-569.

Chen Y, Su W, Yang S, Gefen A, Lin F (2013) In situ forming hydrogels composed of oxidized high molecular weight hyaluronic acid and gelatin for nucleus pulposus regeneration. Acta Biomater 9: 5181-5193.

Choi H, Johnson ZI, Risbud MV (2015) Understanding nucleus pulposus cell phenotype: a prerequisite for stem cell based therapies to treat intervertebral disc degeneration. Curr Stem Cell Res Ther 10: 307-316.
Chung C, Erickson IE, Mauck RL, Burdick JA (2008) Differential behavior of auricular and articular chondrocytes in hyaluronic acid hydrogels. Tissue Eng Part A 14: 1121-1131.

Feng G, Li L, Liu H, Song Y, Huang F, Tu C, Shen B, Gong Q, Li T, Liu L, Zeng J, Kong Q, Yi M, Gupte M, Ma PX, Pei F (2013) Hypoxia differentially regulates human nucleus pulposus and annulus fibrosus cell extracellular matrix production in 3D scaffolds. Osteoarthritis Cartilage 21: 582-588.

Fornes O, Castro-Mondragon JA, Khan A, van der Lee R, Zhang X, Richmond PA, Modi BP, Correard S, Gheorghe M, Baranasic D, Santana-Garcia W, Tan G, Cheneby J, Ballester B, Parcy F, Sandelin A, Lenhard B, Wasserman WW, Mathelier A (2020) JASPAR 2020: update of the open-access database of transcription factor binding profiles. Nucleic Acids Res 48: D87-D92.

Fujita N, Chiba K, Shapiro IM, Risbud MV (2012) HIF- $1 \alpha$ and HIF-2 $\alpha$ degradation is differentially regulated in nucleus pulposus cells of the intervertebral disc. J Bone Miner Res 27: 401-412.

Fujita N, Hirose Y, Tran CM, Chiba K, Miyamoto T, Toyama Y, Shapiro IM, Risbud MV (2014) HIF1-PHD2 axis controls expression of syndecan 4 in nucleus pulposus cells. FASEB J 28: 2455-2465.

Gao MM, Su QN, Liang TZ, Ma JX, Stoddart MJ, Richards RG, Zhou ZY, Zou XN (2018) Transcriptional activation of ENPP1 by osterix in osteoblasts and osteocytes. Eur Cell Mater 36: 1-14.

Ichimaru S, Nakagawa S, Arai Y, Kishida T, ShinYa M, Honjo K, Tsuchida S, Inoue $H$, Fujiwara $H$, Shimomura S, Mazda O, Kubo T (2016) Hypoxia potentiates anabolic effects of exogenous hyaluronic acid in rat articular cartilage. Int J Mol Sci 17: 1013. DOI: 10.3390/ijms17071013.

Isa IL, Srivastava A, Tiernan D, Owens P, Rooney P, Dockery P, Pandit A (2015) Hyaluronic acid based hydrogels attenuate inflammatory receptors and neurotrophins in interleukin-1 $\beta$ induced inflammation model of nucleus pulposus cells. Biomacromolecules 16: 1714-1725.

Ishida O, Tanaka Y, Morimoto I, Takigawa M, Eto S (1997) Chondrocytes are regulated by cellular adhesion through CD44 and hyaluronic acid pathway. J Bone Miner Res 12: 1657-1663.

Jensen EC (2013) Quantitative analysis of histological staining and fluorescence using ImageJ. Anat Rec (Hoboken) 296: 378-381.

Kazezian Z, Li Z, Alini M, Grad S, Pandit A (2017) Injectable hyaluronic acid down-regulates interferon signaling molecules, IGFBP3 and IFIT3 in the bovine intervertebral disc. Acta Biomater 52: 118-129.

Kim DH, Martin JT, Elliott DM, Smith LJ, Mauck RL (2015) Phenotypic stability, matrix elaboration and functional maturation of nucleus pulposus cells encapsulated in photocrosslinkable hyaluronic acid hydrogels. Acta Biomater 12: 21-29.

Krishnamachary B, Penet MF, Nimmagadda S, Mironchik Y, Raman V, Solaiyappan M, Semenza GL, Pomper MG, Bhujwalla ZM (2012) Hypoxia regulates 
CD44 and its variant isoforms through HIF-1 $\alpha$ in triple negative breast cancer. PLoS One 7: e44078. DOI: 10.1371/journal.pone.0044078.

Liang G, Li S, Du W, Ke Q, Cai J, Yang J (2017) Hypoxia regulates CD44 expression via hypoxiainducible factor-1 in human gastric cancer cells. Oncol Lett 13: 967-972.

Liu Z, Zheng Z, Qi J, Wang J, Zhou Q, Hu F, Liang J, Li C, Zhang W, Zhang X (2018) CD24 identifies nucleus pulposus progenitors/notochordal cells for disc regeneration. J Biol Eng 12: 35. DOI: 10.1186/ s13036-018-0129-0.

Mohd II, Abbah SA, Kilcoyne M, Sakai D, Dockery P, Finn DP, Pandit A (2018) Implantation of hyaluronic acid hydrogel prevents the pain phenotype in a rat model of intervertebral disc injury. Sci Adv 4: eaaq0597. DOI: 10.1126/sciadv.aaq0597.

Peroglio M, Eglin D, Benneker LM, Alini M, Grad S (2013) Thermoreversible hyaluronan-based hydrogel supports in vitro and ex vivo disc-like differentiation of human mesenchymal stem cells. Spine J 13: 16271639.

Pfirrmann CW, Metzdorf A, Zanetti M, Hodler J, Boos N (2001) Magnetic resonance classification of lumbar intervertebral disc degeneration. Spine (Phila Pa 1976) 26: 1873-1878.

Pietras A, Katz AM, Ekstrom EJ, Wee B, Halliday JJ, Pitter KL, Werbeck JL, Amankulor NM, Huse JT, Holland EC (2014) Osteopontin-CD44 signaling in the glioma perivascular niche enhances cancer stem cell phenotypes and promotes aggressive tumor growth. Cell Stem Cell 14: 357-369.

Ponta H, Sherman L, Herrlich PA (2003) CD44: from adhesion molecules to signalling regulators. Nat Rev Mol Cell Biol 4: 33-45.

Quero L, Klawitter M, Schmaus A, Rothley M, Sleeman J, Tiaden AN, Klasen J, Boos N, Hottiger MO, Wuertz K, Richards PJ (2013) Hyaluronic acid fragments enhance the inflammatory and catabolic response in human intervertebral disc cells through modulation of toll-like receptor 2 signalling pathways. Arthritis Res Ther 15: R94. DOI: 10.1186/ ar4274.

Rayahin JE, Buhrman JS, Zhang Y, Koh TJ, Gemeinhart RA (2015) High and low molecular weight hyaluronic acid differentially influence macrophage activation. ACS Biomater Sci Eng 1: 481-493.

Risbud MV, Guttapalli A, Stokes DG, Hawkins D, Danielson KG, Schaer TP, Albert TJ, Shapiro IM (2006) Nucleus pulposus cells express HIF-1 alpha under normoxic culture conditions: a metabolic adaptation to the intervertebral disc microenvironment. J Cell Biochem 98: 152-159.

Semenza GL (2000) HIF-1: mediator of physiological and pathophysiological responses to hypoxia. J Appl Physiol (1985) 88: 1474-1480.

Snetkov P,ZakharovaK, MorozkinaS, Olekhnovich R, Uspenskaya M (2020) Hyaluronic acid: the influence of molecular weight on structural, physical, physico-chemical, and degradable properties of biopolymer. Polymers (Basel) 12: 1800. DOI: 10.3390/ polym 12081800

Stevens JW, Kurriger GL, Carter AS, Maynard JA (2000) CD44 expression in the developing and growing rat intervertebral disc. Dev Dyn 219: 381-390.

Stone JE, Akhtar N, Botchway S, Pennock CA (1994) Interaction of 1,9-dimethylmethylene blue with glycosaminoglycans. Ann Clin Biochem 31: 147-152.

Takahashi K, Aoki Y, Ohtori S (2008) Resolving discogenic pain. Eur Spine J 17 Suppl 4: 428-431.

Tran CM, Fujita N, Huang BL, Ong JR, Lyons KM, Shapiro IM, Risbud MV (2013) Hypoxia-inducible factor (HIF)-1 $\alpha$ and CCN2 form a regulatory circuit in hypoxic nucleus pulposus cells: CCN2 suppresses HIF-1 $\alpha$ level and transcriptional activity. J Biol Chem 288: 12654-12666.

Vergroesen PP, Kingma I, Emanuel KS, Hoogendoorn RJ, Welting TJ, van Royen BJ, van Dieen JH, Smit TH (2015) Mechanics and biology in intervertebral disc degeneration: a vicious circle. Osteoarthritis Cartilage 23: 1057-1070.

Zhou Z, Gao M, Wei F, Liang J, Deng W, Dai X, Zhou G, Zou X (2014) Shock absorbing function study on denucleated intervertebral disc with or without hydrogel injection through static and dynamic biomechanical tests in vitro. Biomed Res Int 2014: 461724. DOI: 10.1155/2014/461724.

\section{Web References}

1. http://jaspar.genereg.net/ [25-08-2020]

2. http://www.cbs.dtu.dk/services/Promoter/ [2508-2020]

3. http://genome.ucsc.edu/cgi-bin/hgGateway [25-08-2020]

Editor's note: There were no questions from reviewers for this paper, therefore there is no Discussion with Reviewers section. The Guest Editor responsible for this paper was Andrea Vernengo. 